\title{
Relaxation Increases Monetary Valuations
}

Michel Tuan Pham, Columbia University

Iris Hung, National University of Singapore

Gerald Gorn, University of Hong Kong*

Whether buying a house, negotiating the price of a car, or considering whether to invest in the stock market, common wisdom holds that people should be relaxed and at ease. Relaxation is among the most important environmental states marketers try to create, whether in spas, hotel rooms, or restaurants. Yet, there is little consumer research on relaxation. Our research documents an intriguing phenomenon: states of relaxation increase consumers' monetary valuations of products, as compared to equally pleasant but less relaxed states. In other words, the more relaxed a consumer is, the more likely it is that they will perceive the service(s) as having monetary value.

While the effects of affective states on consumer behavior have received a considerable amount of attention, previous research has mostly focused on the effects of positive versus negative overall moods (Pham, 1998; Yeung \& Wyer, 2004), and to a lesser extent the effects of emotional arousal (Pham, 1996), and more recently the effects of specific negative emotions like anger, anxiety, sadness, and disgust (Raghunathan, Pham, \& Corfman 2006). Only a few studies have directly focused on the effects of relaxation. Gorn and colleagues (1997) found that the use of relaxing, "whitish," pastel-like background colors in print ads resulted in more favorable evaluations of advertised products than the use of non-relaxing colors with a "blackish" quality in them (e.g., a dark brown; see also Gorn, Chattopadhyay, Sengupta \& Tripathi, 2004). While Gorn and colleagues (1997, 2004) examined the effects of relaxation compared to non-relaxation, more recent research has examined the effects of relaxation compared to other positive affective states such as cheerfulness and excitement (Bosmans \& Baumgartner, 2005).

The present research investigates a very different phenomenon: the effects of relaxation on consumers' monetary valuations of products. Our research suggests that relaxation may actually increase consumers' monetary valuations of products for the following reason: states of relaxation seem to encourage a broader and more contemplative form of thinking (Fredrickson \& Branigan, 2005). Applied to the context of our research question, we propose that relaxed consumers have more global and abstract representations when assessing the monetary value of products than non-relaxed consumers. Research (Liberman \& Trope, 1998) shows that individuals who have more abstract representations (higher-level construals) of goal-directed activities (e.g., going on a vacation in Mexico) tend to focus more on the overall desirability of these activities (e.g., how appealing Mexico is), whereas individuals who have more concrete representations (lower-level construals) tend to focus more on the feasibility of these activities (e.g., the cost, the amount of travel involved). Linking these two streams of research together, it would be reasonable to infer that relaxation would encourage a more abstract representation (or higher-level construal) of the value of products, which would shift a consumers' focus towards the general desirability of these products and away from their more concrete characteristics. Because most consumer products (e.g., vacations, cameras) are inherently desirable, such a shift in representation would result in the products being valued more by relaxed consumers than by less-relaxed consumers. For example, in assessing the monetary value of a digital camera, a relaxed consumer would focus more on the overall desirability of owning the camera (e.g., the pictures it will enable them to take and the memories they will be able to collect) and less on the concrete features of the camera itself (e.g., the number of megapixels, weight, shutter speed). This shift in representation should result in relaxed consumers perceiving the camera to be more valuable than less-relaxed consumers.

Across four studies, more than 450 participants were induced into either a state of relaxation, or into an equally pleasant (but less relaxed) affective state. This was done with the use of appropriate, pre-tested videos. Next, as part of a supposedly unrelated study, they were asked to assess the monetary value of products. Different measures of monetary valuation were used across different studies. The results consistently show that participants who were more relaxed assigned greater monetary value to the products than participants who were less relaxed. This phenomenon is likely not due to the pleasantness of relaxation itself because the conditions were matched in terms of pleasantness (which was verified by confounding checks).

The first study demonstrated the basic phenomenon across various product categories. On average, participants in the more relaxed condition assigned higher monetary valuations than participants in the less relaxed condition to products supposedly from a product catalogue. Using a scenario where participants were asked to imagine that they were interested in a digital camera available on eBay, the rest of the studies replicated this effect using more refined measures of monetary valuation: (1) the maximum amount of money that participants were willing to bid for the camera; and (2) participants' estimates of how much the camera was really worth. Study 2 found that while relaxation influenced overall measures of monetary valuation, it did not affect more specific ratings of product perception. This was presumably because specific ratings of product perceptions trigger a more concrete level of representation of the product's value. Our reasoning was confirmed in Studies 3 and 4, with Study 4 providing direct support for this explanation by directly manipulating participants' levels of representation through a priming procedure. If the effect of relaxation on monetary valuation is due to higher levels of representation, then the priming of an abstract level of thinking should amplify this effect by reinforcing relaxed individuals' tendency to represent the value of products at a higher level. Conversely, the priming of a lower level of construal should attenuate this effect by inhibiting relaxed individuals' tendency to represent products at a higher level. The results were consistent with this reasoning.

Overall, the evidence suggested that relaxed individuals inflate the monetary value of products. The bids of less-relaxed participants in the bidding experiments were generally close to the market value of the product. Relaxed participants' estimates were however, substantially higher than the market value of the product, unless high-level construals were discouraged by the manipulations. Even though it is typically not in people's material 
interest to have inflated perceptions of the monetary value of products they might buy, our evidence suggests that, nonetheless, relaxed consumers do have inflated perceptions.

For further information contact: Gerald Gorn, University of Hong Kong, mkgorn@ust.hk

\title{
Arousal and Subjective Probabilities: An Alternative Interpretation of Wishful Thinking
}

\author{
Joachim Vosgerau, Carnegie Mellon University*
}

A wealth of psychological research indicates that people are unrealistically optimistic about future outcomes. Overoptimism about future outcomes is called desirability bias (i.e., the tendency to judge desirable outcomes as more likely than undesirable outcomes, Krizan and Windschitl 2007). For example, in the classic study by Crandall, Solomon, and Kellaway (1955), participants were asked to predict random independent draws from decks of ten cards. Even though they knew that each deck contained 7 winning cards, they predicted drawing a winning card $89 \%$ of the time. While such findings are typically interpreted as people suffering from a desirability bias, they actually don't show desirability bias at all. Knowing that the objective probability of a winning card is 70\%, a rational decision maker would predict a winning card in $100 \%$ of the draws (thereby maximizing accuracy of prediction). So, compared to a rational decision maker, participants in the above experiment appear to be pessimistic or at least not accuracy-maximizing. Whatever is the case, they are certainly not overoptimistic.

A better measure for optimism and pessimism than outcome predictions is likelihood judgments (cf., Bar-Hillel, Budescu, and Amar 2008). I propose that the likelihood of an event may be judged by how aroused the stake-holder is. Misattribution of arousal has been demonstrated for a broad range of emotional states and judgments such as fear, anger, sexual excitation, attractiveness, funniness of cartoons (Reisenzein, 1983), and judgments of heights (Stefanucci \& Storbeck, 2009). I hypothesize that the greater the stake in the outcome (e.g. winning $\$ 5$ or losing $\$ 5$ if event occurs vs. neutral outcome), the more aroused will the stake-holder be, and the higher s/he will judge the likelihood of the outcome occurring or not occurring. In study 1, I tested whether likelihood judgments can be misattributed to arousal. The study employed a 2 (arousal vs. no arousal) $\times 2$ (probability of occurrence vs. non-occurrence) between-subjects design. Half of the participants were asked questions such as "How likely do you think the Yankees will win the World Series?"; the other half was asked for the complementary probabilities. Arousal was manipulated by printing questionnaires on either pink or grey paper. As hypothesized, participants judged both outcomes (the Yankees winning and the Yankees not winning) as more likely in the arousal condition (pink paper).

Study 2 tested misattribution of arousal by manipulating the order in which arousal was rated and likelihood judgments were made. Half of the participants were first asked to rate their arousal level, and subsequently to judge the likelihood of outcome occurrence or non-occurrence (outcome: participants won $\$ 5$ when they got at least one 3 within four die-rolls, $p=51.77 \%$ ). The other half was first asked to judge the likelihood of outcome occurrence/non-occurrence, and then to rate their arousal level. As hypothesized, participants reported higher arousal before than after the likelihood measures, and rated the likelihood of the outcome occurring/non-occurring as higher before than after the arousal measures.

In study 3 I tested whether arousal is higher for both, negative and positive stakes in the outcome than neutral outcomes. Accordingly, probability of occurrence and non-occurrence should be judged as more likely when participants have a stake in the outcome (winning or losing a shot-glass) than when they have no stake in the outcome. The study employed a 2 (probability of outcome occurrence vs. non-occurrence) $\times 3$ (stake in outcome: win a shot-glass vs. neutral vs. lose a shot-glass) between-subjects design. Half of the participants was asked how likely a computer, when rolling a die four times, would roll a 6 twice. The other half was asked how likely the computer would not roll a 6 twice. Participants earned $\$ 1$ if their probability estimate was within the $10 \%$ range of the objective probability $(p=11.57 \%)$. In the win condition, participants won a shot-glass when the computer rolled a 6 twice, in the neutral condition no gain or loss was associated with outcome occurrence, and in the lose condition participants were first endowed with the shot-glass and lost it when the computer rolled a 6 twice. As hypothesized, participants judged the probability of getting a 6 twice higher in the win $(M=19.77 \%)$ and the lose condition $(M=38.91 \%)$ than the neutral condition $(M=13.44 \%)$, and judged the probability of not getting a 6 twice also higher in the win $(M=72.32 \%)$ and the lose condition $(M=61.79 \%)$ than the neutral condition $(M=55.83 \%)$.

Finally, in study 4 German soccer fans were asked to imagine watching an upcoming match either live (more aroused) or taped (less aroused; having the prospect of watching a match live is more arousing than having the prospect of watching it taped; Vosgerau et al. 2006, JCR). Fans were also asked to indicate how likely their team (Stuttgart) was to win. When having the prospect of watching the match taped, Stuttgart was judged as equally likely to win/tie $(M=52.40 \%)$ as to lose $(M=51.36 \%)$. In contrast, for the live broadcast conditions, Stuttgart was rated as more likely to win/draw $(M=58.48 \%)$ and also as more likely to lose $(M=67.92 \%)$. Taken together, the four studies demonstrate that arousal (from having a stake in the outcome) can make consumers more optimistic and more pessimistic. Such optimism and pessimism call into question the ubiquity of wishful thinking and general over-optimism.

For further information contact: Joachim Vosgerau, Carnegie Mellon University, vosgerau@andrew.cmu.edu 